\title{
Napoléon et l'Europe
}

Le point de vue anglo-américain

Michael Broers, Steven Englund, Michael Rowe et Annie Jourdan

\section{(2) OpenEdition}

Journals

Édition électronique

URL : https://journals.openedition.org/ahrf/11000

DOI : 10.4000/ahrf.11000

ISSN : 1952-403X

Éditeur :

Armand Colin, Société des études robespierristes

Édition imprimée

Date de publication : 1 décembre 2008

Pagination : 131-153

ISSN : 0003-4436

Référence électronique

Michael Broers, Steven Englund, Michael Rowe et Annie Jourdan, « Napoléon et l'Europe », Annales historiques de la Révolution française [En ligne], 354 | octobre-décembre 2008, mis en ligne le 01 décembre 2011, consulté le 22 avril 2022. URL : http://journals.openedition.org/ahrf/11000;DOI : https://doi.org/10.4000/ahrf.11000 


\title{
NAPOLÉON ET L'EUROPE \\ LE POINT DE VUE ANGLO-AMÉRICAIN
}

\author{
Michael BROERS \\ Steven ENGLUND \\ Michael ROWE \\ Annie JOURDAN
}

\begin{abstract}
Aborder « Napoléon et l'Europe » en donnant la parole à trois historiens anglo-américains pourra paraître surprenant aux lecteurs des $A H R F$. La motivation à la base de cette sélection est pourtant évidente. Ces dernières années, les recherches les plus novatrices sur ce sujet précis émanent du monde anglo-saxon, non seulement parce que leurs auteurs ont renouvelé les questionnements et les approches, mais encore parce que leur point de vue est plus distancié que ne saurait l'être celui des historiens français. C'est que les chercheurs anglo-américains ont pour immense avantage de ne pas récrire leur propre histoire nationale, celle qui les a formés. Ils sont confrontés à deux réalités étrangères - leur(s) pays d'investigation et la politique napoléonienne dans ce(s) même(s) pays -, ce qui leur permet justement de prendre leurs distances vis-à-vis de leur objet.

Le père fondateur de cette école nouvelle est sans nul doute Stuart Woolf, dont le Napoléon et la conquête de l'Europe (Flammarion, 1990) a du reste été publié tout d'abord en français. Michael Broers a suivi avec un retentissant Europe under Napoleon (Londres, 1996) et Europe after Napoleon (Manchester, 1996). Tous deux sont à l'origine du renouveau d'intérêt anglo-américain (voire australien) pour la période napoléonienne, notamment dans ce qui a trait à son impact en Europe. Depuis, en effet, les régions et les États du continent ont été étudiés cas par cas : Michael Rowe s'est intéressé à la Rhénanie; John Davis au royaume de Naples ; Charles Esdaile et John Tone à l'Espagne; David Laven à la Vénétie; Alexander Grab à l'Italie septentrionale, etc. Ces historiens transnatio-
\end{abstract}


naux plus que nationaux ont vécu dans le pays qu'ils décrivent et en ont découvert de près les spécificités; ils en ont étudié les archives et les sources, tout en ne négligeant pas les archives françaises. C'est dans ces ouvrages pluriculturels que se décèle, me semble-t-il, un vrai renouvellement des études sur le Premier Empire. Les recueils d'articles publiés à la suite de colloques sont tout aussi stimulants : Collaboration and Resistance in Napoleonic Europe. State-Formation in an Age of Upheavals (éd. M. Rowe, Londres, 2003) l'illustre plutôt bien, quand il brosse un tableau tout en nuances de l'Europe de Napoléon.

Ce n'est certes pas dire que la France serait dénuée de grands chercheurs en la matière, mais là, les avancées touchent plus aux études sur Napoléon en tant qu'empereur des Français : ainsi, la Nouvelle histoire du Premier Empire de Thierry Lentz, les recherches de Natalie Petiteau sur le mythe napoléonien, la noblesse d'empire ou les vétérans; la thèse d'Igor Moullier sur le ministère de l'Intérieur ou celle à paraître de Cyril Triolaire sur les divertissements consulaires et impériaux, pour ne citer que quelques exemples fort diversifiés : tous ces travaux démontrent que les études napoléoniennes y sont en net progrès. Mais dans ce domaine aussi, les travaux des Anglo-Américains leur font concurrence : ceux de Michael Sibalis sur les commissions sénatoriales pour les libertés; de Jeff Horn sur les élections et de Rafe Blaufarb sur les continuités entre Ancien Régime et Empire ou bien encore ceux de Jennifer Heuer sur les relations familiales en période de guerre.

Pour ce qui est de l'Europe, aucun doute, ce sont les monographies anglo-américaines qui l'emportent, en ce qu'elles sont remarquablement bien informées et qu'elles proposent des vues approfondies sur les expériences et les attentes dans les pays concernés. Ici, il est vrai que les « nationaux » leur font à leur tour concurrence : en Allemagne, en Italie, en Espagne ou aux Pays-Bas, voire en Suisse, en Pologne ou en Russie, de jeunes chercheurs se réintéressent à une période jadis ignorée par leur tradition historique respective, précisément parce qu'elle était considérée comme «non nationale ». Les chercheurs anglo-saxons leur servent en un sens de porte-parole ou de relais et popularisent des travaux inaccessibles à un public qui ne connaît pas forcément leur langue. Les trois intervenants de ces regards croisés notent bien du reste le problème linguistique pour quiconque voudrait s'atteler à une histoire vraiment européenne. Ce n'est là qu'un des obstacles à une pleine appréhension du sujet. Un autre réside dans l'incompréhension mutuelle entre historiens français et angloaméricains. Laisser la parole à ces derniers pourrait faciliter le dialogue. De là ces regards croisés. 


\section{Annie Jourdan}

1. Ce qui me frappe actuellement, c'est l'insistance quasi-essentialiste des historiens anglo-américains sur l'origine nationale de leurs collègues, notamment français, mais aussi anglais; sur leurs traditions intellectuelles et les écoles historiques auxquelles ils appartiennent. Cette tendance ne risque-t-elle pas de mener à des généralisations abusives et d'amplifier l'incompréhension mutuelle?

\section{Steven ENGLUND}

Le problème de l'estime et du mépris dans l'historiographie anglo/ américaine-française n'est qu'un volet fascinant d'un plus vaste diptyque touchant à la question de la compréhension ou de l'incompréhension et de la rivalité anglo-française en général - ou faut-il dire " franco-anglaise » ? Trop vaste, en vérité, pour que cette question soit traitée en quelques paragraphes. Les relations scientifiques anglo-françaises ont leur propre histoire. Pendant de longues années, les universitaires français ont fait preuve d'une superbe souveraine envers leurs collègues étrangers (en particulier, ceux qui écrivaient en anglais), et à l'exception des « happy few » (Richard Cobb, Eugen Weber, etc.). Depuis les années 1970, tout cela a changé - à tel point que, désormais, le paysage est quasiment inversé. Michael Rowe, par exemple, cite relativement peu d'auteurs français parmi ses très nombreuses références. Je connais bien des historiens français du Premier Empire qui trouveraient cela aberrant. En vérité, j'en connais dans le monde scientifique français qui trouvent aberrant que, depuis quelque temps, leur histoire échappe pour une grande part à leur contrôle «national». Du point de vue quantitatif, il y a sans doute tout autant, sinon plus, d'AngloAméricains qu'il n'y a de Français pour étudier l'histoire de France. Et dans quelques domaines, comme le Premier Empire, les étrangers actuellement se trouvent faire des recherches plus pointues et ouvrir des réflexions plus novatrices que leurs collègues français.

D'autre part, je ne doute pas que Michael Rowe ne s'empresse d'expliquer que son désintérêt pour les ouvrages français n'a rien d'une superbe ou d'un " essentialisme » national à leur endroit. C'est tout simplement qu'il n'y a aucun chercheur français récent à avoir entrepris des recherches ou à avoir émis des réflexions sur la question du Premier Empire en Allemagne. Les Français se focalisent sur « la France de Napoléon », alors que les Anglo-Américains se concentrent sur « l'Empire ». Actuellement, il y a tout un réseau d'institutions consacrées à l'étude du Premier Empire en tant que phénomène européen - entre autres, les congrès en cours (parrainés par des historiens anglo-allemands), intitulés 
« Experience, Memory and Media : Transmitting the Revolutionary and Napoleonic Wars in $19^{\text {th }}$ and $20^{\text {th }}$ Century Europe $»$, congrès qui se tiennent dans des villes variées, le plus récent ayant eu lieu à Mannheim, en octobre dernier. Détail frappant: les deux historiens français éminents qui y étaient invités se sont désistés au dernier moment.

$\mathrm{Au}$ fond, alors que les universitaires français citent plus que jamais des sources étrangères (surtout anglophones), ils ne font aucun effort pour entamer des discussions avec les auteurs de ces travaux et leurs interprétations. La Fondation Napoléon a remis récemment le prix du meilleur livre étranger à Michael Broers, mais j'ai été frappé de voir que peu de membres du jury avaient lu ou compris la thèse de ce livre, Napoleonic Empire in Italy. Je suis heureux que Broers ait eu ce prix bien mérité, mais je crains que rares soient ceux dans le monde français de l'histoire napoléonienne qui fassent l'effort de comprendre son point de vue global. Cela vient pour une part de ce que les livres ne font pas en France l'objet de véritables débats. Il n'y a pas d'équivalent à H-France et les comptes rendus scientifiques sont rarement critiques.

Enfin, sans vouloir sur ce point être trop « Noirielien » ou « Bourdieusien », le désintérêt réciproque des historiens anglo-français est dû pour beaucoup à une sociologie de base : les deux groupes écrivent dans leur propre langue (nationale) pour leur propre public (national); sont payés par leurs institutions (nationales) respectives et adhèrent aux us et coutumes (nationaux) de leur monde académique. Remarquable en ce qui concerne le dernier point est le fait qu'il y a moins de différence en France entre la monographie scientifique et la synthèse grand public. En d'autres termes, la République des Lettres, censée être internationale et cosmopolite, vit seulement dans l'esprit de quelques idéalistes. Sinon, les historiens sont pour beaucoup d'entre eux de simples universitaires, réagissant principalement aux récompenses et aux sanctions nationales.

\section{Michael Broers}

Une des raisons de ces malentendus est peut-être l'éclipse de l'histoire marxiste, qui a réduit l'ampleur des débats en France même, où elle tenait une place très importante. L'autre, quoi qu'il en soit, est la tendance générale parmi les historiens français à concentrer leurs travaux sur la France de Napoléon. Cette différence d'approche s'est amplifiée, alors que les Anglo-Saxons ont modifié leurs perspectives. Vous avez raison cependant de nous rappeler que cette différence d'approche de la période ne doit pas mener à en conclure à une uniformité de perspective (francocentriste), doublée d'une uniformité d'opinion (elle aussi francocentriste). 


\section{Michael Rowe}

Les historiens de la période révolutionnaire et napoléonienne ont tout d'abord la formidable chance d'explorer des événements et des processus qui ont une dimension vraiment paneuropéenne. Pour exemples, les guerres qui ont marqué la période, de même que les processus de mobilisation de ressources (conscription, fiscalité, etc.) ont été une expérience partagée par la grande majorité des Européens. Aussi, les historiens de la période devraient-ils en principe être bien placés pour s'élever audessus des traditions nationales auxquelles ils appartiennent. Après tout, chaque spécialiste de cette période prenant part aux conférences et congrès qui se tiennent annuellement sur des thèmes napoléoniens est confronté à des historiens du continent et bien au-delà.

Néanmoins, alors que les guerres napoléoniennes ont été une expérience partagée au niveau paneuropéen, elles - ou plutôt leur mémoire - ont fortement contribué au développement du nationalisme du dix-neuvième siècle. L'émergence de systèmes nationaux d'éducation et d'historiographies nationales a nourri ce processus, dont nous ressentons aujourd'hui encore les effets.

Nulle part peut-être cela est plus vrai que dans l'historiographie de la région à laquelle je me suis particulièrement intéressé, à savoir la Rhénanie. Les récits sur les expériences de cette région durant l'époque française étaient inextricablement liés aux vicissitudes des relations franco-germaniques des $\mathrm{XIX}^{\mathrm{e}}$ et $\mathrm{XX}^{\mathrm{e}}$ siècles. Une des conséquences en a été la publication relativement importante d'études sur ce qu'était et ce qu'est une petite région. La plupart du reste étaient des pamphlets de propagande politique plus que des études scientifiques sérieuses, surtout au moment de la détérioration des relations franco-allemandes qui a suivi la Première Guerre mondiale.

Évidemment, on pourrait avoir l'audace de croire qu'un nationalisme aussi vif est désormais mort et enterré. L'historiographie napoléonienne rhénane des années post-soixante semble l'indiquer. Mais cela ne veut pas dire que les préoccupations politiques contemporaines se soient évanouies. Car, dans notre désir d'éviter le nationalisme ancien, nous risquons d'ignorer des questions relatives à la collaboration, à la résistance et à l'identité nationale durant l'époque napoléonienne au profit de thèmes qui semblent moins aptes à raviver les anciens conflits. Pis. On risque de tomber dans le piège qui consisterait à donner une légitimité historique à l'intégration européenne actuelle en antidatant le processus de deux siècles. Les historiens non-français et non-allemands de la Rhénanie des années 1970 et 1980 - comme T.C.W. Blanning - sont, en tant qu'« outsi- 
ders » mieux placés en un sens pour focaliser sur les conflits nationaux que les historiens français ou allemands, auxquels on serait vite enclin de reprocher ce type d'approche.

\section{Annie Jourdan}

2. Les écoles historiques se différencient en effet les unes des autres et elles évoluent dans le temps. Aux États-Unis comme en Grande-Bretagne, il est de plus en plus d'usage de poser une grande thèse a priori, ce qui incite les historiens à ne rechercher et à ne noter que ce qui entre dans leur schème. Que pensez-vous de cette tendance? Est-elle la cause du peu de traductions d'ouvrages français en anglais? Car, s'il est une autre tendance, c'est bien en effet la rareté des ouvrages traduits en anglais.

\section{Michael Broers}

Pour ce qui est de la traduction : les éditeurs ne le font pas parce que c'est trop coûteux. Tous les historiens anglophones, pourtant, j'en suis sûr, souhaiteraient avoir plus de travaux traduits en anglais. En tant qu'impliqué intensivement dans l'édition et au courant de ses pratiques, je refuse d'en attribuer la responsabilité aux universitaires. Et puis, l'historiographie française pèse moins lourd qu'auparavant, cela vient de ce qu'elle est devenue un peu convenue et qu'elle se limite à la France dans nombre de cas, et, enfin, qu'elle ne participe pas vraiment aux nouveaux débats. Heureusement, de plus en plus de Français lisent leurs homologues anglo-saxons - entre autres, Thierry Lentz - et cela peut faire bouger les choses et avoir des conséquences importantes dans l'avenir.

En ce qui concerne la " grande thèse », elle peut s'appliquer à plusieurs d'entre nous - à moi, en particulier ou bien à Esdaile. Mais le marxisme français a joué un grand rôle dans notre formation. Je ne crois pas que cela vaille pour les travaux de Michael Rowe ou d'Alex Grab.

\section{Michael Rowe}

Sur cette préférence donnée à la grande thèse, je ne suis pas sûr que ce soit vrai. Dans les années 1980, n'oubliez pas que les historiens marxistes étudiant la République de Mayence rejetaient plutôt dédaigneusement les historiens britanniques qui travaillaient sur le même sujet, sous prétexte qu'ils n'avaient pas une vaste vue (marxiste) d'ensemble ou au contraire, parce qu'ils faisaient du « fétichisme archivistique ».

Mais sans doute faut-il ici noter des facteurs structuraux autant qu'idéologiques, quand on commente ce genre de différences auxquelles se réfère la question : le fait que le monde de l'édition anglo-saxon (que 
mes collègues celtiques m'excusent pour l'usage de ce terme) est plus commercial. Là, des pressions sont exercées sur l'auteur pour qu'il produise pour un marché aussi vaste que possible. Il y a deux façons de le faire : élargir le thème autant que possible ou bien relier la recherche à une controverse contemporaine : telle que l'intégration européenne ou les guerres en Afghanistan et en Irak.

Pour ce qui est des traductions des ouvrages français ou non anglosaxons, c'est plus que regrettable. Et c'est particulièrement vrai pour les ouvrages allemands sur la période qui nous concerne. Mais c'est moins parce qu'il y a un manque d'intérêt chez les spécialistes - eux lisent du reste la version originale - qu'à cause des intérêts économiques du marché du livre. Les livres spécialisés en histoire allemande, s'ils ne parlent pas des nazis, n'ont pas un grand lectorat - ce qui n'est pas fait pour justifier le surplus des frais de traduction.

\section{Steven Englund}

Je ne suis pas non plus d'accord que la grande thèse a priori soit avant tout une marotte anglo-américaine. Daniel Mornet, Fernand Braudel et Ernest Labrousse ne sont-ils pas des Français ? Et leurs chefs-d'œuvre ne sont-ils pas élaborés autour de thèses a priori? Et ceux de Soboul ou de Furet? Et que dire de Jean-Paul Bertaud, qui vient de nous donner un ouvrage à thèse sur le Premier Empire (thèse très critique de l'Empire, par ailleurs) ? C'est un excellent ouvrage que j'espère voir paraître en anglais. Par contre, Rafe Blaufarb et Jeff Horn sont des historiens du Premier Empire qui écrivent des monographies utiles, et non des essais à thèse ou avec de grands a priori. Qui plus est, ils privilégient la France elle-même et non l'Empire, parce que, contrairement à leurs collègues, ils ne pensent pas qu'elle ait été trop étudiée au détriment des provinces. Autre ouvrage à mériter une traduction : la biographie de Talleyrand d'Emmanuel de Waresquiel, qui est un des plus beaux ouvrages écrits sur cette période, pour lequel j'essaie de trouver un éditeur américain, mais ce n'est pas facile pour des raisons qui n'ont rien à voir avec l'essentialisme nationaliste ou les préjugés, mais tout simplement avec le capitalisme. Entre-temps, pour les raisons citées plus haut, des éditeurs anglais ou américains peuvent très bien commander des biographies plus modestes qui - par nécessité - vont piller le superbe chef-d'œuvre de Waresquiel.

\section{Annie Jourdan}

3. Il n'est pas question d'affirmer que l'absence de traductions en anglais provient d'un essentialisme nationaliste; je voulais seulement dire que les comptes rendus anglo-américains (de $\mathrm{H}$-France pour préciser) situent 
parfois l'auteur et ses réflexions dans une tradition nationale ou historique. Dernièrement, j'étais moi-même étiquetée de "gaulliste 》-puisque d'origine française et nonobstant mon allergie aux grands hommes - et Peter Campbell était péremptoirement cantonné dans une tradition britannique: comme si nos origines devaient nécessairement biaiser nos recherches.

Pour ce qui est de Napoléon et l'Europe, les études récentes, surtout anglo-saxonnes du reste, ont amplement traité les États qui constituent aujourd'hui l'Italie et l'Allemagne. Il n'en va pas de même de la Belgique, des Provinces-Unies, de la Suisse, de la Pologne ou de l'Europe orientale. Tant que les expériences " impériales » de ces pays seront méconnues, il est difficile de conclure quoi que ce soit sur l'Europe napoléonienne. Et de ce fait, les grandes synthèses de Stuart Woolf et de Michael Broers sont-elles vraiment fiables? Enfin, que pensez-vous qu'il reste à faire pour mieux comprendre l'Europe « impériale»? Quels travaux privilégieriez-vous?

\section{Michael Broers}

Affirmer que certains pays auraient été négligés est tout à fait injuste. Woolf et moi, nous avons abordé les Provinces-Unies, la Pologne ou la Hongrie, de façon certes limitée à cause des contraintes éditoriales. Woolf et moi, nous avons aussi été les premiers ou presque à prendre en considération un pays comme l'Illyrie. Il est sûr qu'il reste encore beaucoup à faire dans presque tous les domaines. Car la période napoléonienne peut se féliciter d'avoir quelques-unes des meilleures archives du monde. Malgré ce que nous avons fait depuis les vingt ou vingt-cinq dernières années, nous sommes loin du compte. Mes propres recherches actuelles portent sur l'introduction du Code civil dans les départements réunis et les royaumes satellites. Et ce, à partir des archives centrales. Je crois que c'est un domaine d'investigation incontournable - jusqu'à présent, surtout étudié par les universitaires allemands - parce que, nous en sommes tous convenus, c'est un des héritages les plus importants et les plus durables $\mathrm{du}$ règne napoléonien, alors que nous en savons relativement peu à ce sujet. Je ressens par ailleurs le besoin de réfléchir plus longuement sur la période en tant qu'expérience impériale (ou plus précisément coloniale) et d'intégrer l'histoire napoléonienne dans celle de l'impérialisme moderne, entre autres parce qu'il s'est avéré que, dans toute l'Europe, nombre de Français occupaient beaucoup de postes au niveau local. Cela demande donc des recherches sur l'histoire sociale de l'Empire - en termes de relations sociales entre occupants et occupés, et pas seulement politiques. Enfin, l'histoire du genre - le gender - tient une place de choix dans la période napoléonienne, moins en termes de répression des femmes 
par le Code civil, qu'en tant qu'expériences de femmes françaises vivant à l'étranger - une jeune génération de femmes accompagnaient leurs maris/amis dans toute l'Europe et participaient à cet impérialisme.

\section{Steven Englund}

Quoi ? Combien d'Anglo-Américains ne sont-ils pas à avoir travaillé sur des régions autres que l'Italie et l'Allemagne ? Esdaile et Tone sur l'Espagne, Schama sur la Hollande, Woolf, Broers, Bundy et Grab sur l'Illyrie, Christopher Blackbourn sur la Pologne. Le problème, c'est la langue. On n'apprend pas si facilement le néerlandais ou le polonais. D'autre part, il est vrai qu'il est aisé de travailler sur ces pays, puisqu'on a aussi accès à des archives en français, soigneusement conservées et centralisées. Je pense que Broers va produire un jour une synthèse majeure sur le Premier Empire, basée sur de vastes recherches d'archives, qui remplacera l'étude classique de Stuart Woolf et en deviendra la meilleure analyse globale. Mais n'oublions pas non plus, sur un autre front (synthétique), les formidables volumes de Lentz qui intègrent les travaux étrangers sur le Premier Empire. Je ne connais personne d'autre que Lentz pour simultanément suivre l'historiographie en anglais, assister aux conférences anglo-américaines et, dans une certaine mesure, participer aux débats anglophones sur l'histoire de l'Empire français. Et puis, honnêtement, j'ai l'impression que les universitaires français ont plus de peine à lire les langues étrangères qu'ils ne le devraient. Ils ont donc en un sens laissé à d'autres les travaux sur la Belgique et la Hollande du Premier Empire, sans parler de la Pologne.

Il est également vrai que bien des historiens commencent à penser qu'exclure la France de l'Empire « interne » et « externe » est tant soit peu exagéré. Heureusement, de jeunes chercheurs comme Jeff Horn, Rafe Blaufarb et Natalie Petiteau, de même que d'autres chercheurs français, ont concentré leurs travaux sur la France métropolitaine à l'époque napoléonienne (ainsi que le fait depuis plus longtemps l'historien bien connu, Alan Forrest). Et puis, il y a la réédition ultra complète de la correspondance de Napoléon gérée par la Fondation Napoléon, de même que le remarquable volume de lettres de Napoléon et de Joseph, publié par l'infatigable jeune chartiste, Vincent Haegele. Il reste néanmoins beaucoup à faire sur le plan provincial, départemental et local. Prenez par exemple l'ouvrage récent de Howard Brown, tout juste couronné par deux prix, Ending the Revolution: Violence, Justice and Repression in France from the Terror to Napoleon (University of Virginia, 2006) : c'est une rare alliance de recherches archivistiques pointues et de réflexions critiques 
sur les idées reçues : notamment celle qui consiste à placer la rupture d'une séquence de l'histoire de France en 1799. L'étude de Brown sur les cours de justice et la répression démontre au contraire que 1802, au cœur même du Consulat, serait une date plus logique de rupture. C'est une thèse qui va à rebrousse-poil d'une certaine idée républicaine.

Chacun de nous a son projet favori pour des recherches futures. Si j'avais un doctorant brillant et énergique, passionné par l'idéologie, la politique et le langage, je l'inciterais à lire méticuleusement le Moniteur universel pour y noter l'évolution de termes comme " Grande Nation », « République », «Empire/République », «Empire » tout court, tels qu'ils ont été utilisés et interprétés par les régimes successifs pour se légitimer. Je pressens que ce qu'il en ressortirait modifierait la ligne de démarcation entre " République » et " Empire », qui est bien plus floue que ne le croient les historiens français (et cela vaut encore plus pour les années entre 1848 et 1880, ainsi que l'a démontré Robert Tombs dans un volume magistral sur la France du dix-neuvième siècle).

\section{Michael Rowe}

Pour être juste, il faut noter que la superficie de l'Italie et de l'Allemagne justifie le nombre élevé de publications, plus que ce ne saurait être le cas pour de petits pays comme la Belgique et les Provinces-Unies. Et puis, Simon Schama - un des rares historiens à être vraiment populaire en Grande-Bretagne - a consacré son premier livre aux Pays-Bas durant cette période. Sans doute, les territoires les plus négligés sont ceux de l'Europe centrale et orientale : la Pologne, la Russie, la monarchie des Habsbourg et les Balkans. Dans les histoires globales de l'Europe, on en tient compte désormais, du moins en Grande-Bretagne. Pour exemples, l'histoire de l'Europe de Norman Davies et celle sur l'Europe du dixneuvième siècle de Michael Rapport.

Une part du défi pour quiconque entreprendrait de rendre compte de la diversité européenne est bien sûr linguistique. Un accès direct aux sources primaires sur un pays comme la Hongrie exige non seulement une connaissance du hongrois mais aussi de l'allemand et du latin (et peut-être même du français). Un historien étranger capable de maîtriser les langues requises, quoi qu'il en soit, préférera sans doute se concentrer sur la période la plus exaltante de l'histoire du pays concerné : l'Âge d'Or hollandais, l'Allemagne de Bismarck, l'Autriche de Joseph II ou la Hongrie de 1848.

En ce qui concerne le Premier Empire, et étant donné la tendance, perceptible chez Broers, par exemple, je suis sûr qu'on en viendra à des comparaisons entre l'empire de Napoléon et des entités expansionnistes 
similaires, comme les États-Unis d'Amérique ou les empires coloniaux européens. De la même façon, les historiens de l'empire britannique John Wilson, par exemple, qui a travaillé sur les Indes de la fin du dixhuitième et du début dix-neuvième - auront envie de comparer ces expériences d'outre-mer avec la construction d'empire(s) en Europe. Ce processus - une rupture des barrières entre l'histoire européenne et impériale (à comprendre comme non-européenne) - est encore peu élaboré, mais bel et bien en germe.

Pour ce qui est des travaux à entreprendre, la réponse est évidente : combler les lacunes géographiques, y compris l'Europe orientale. L'image qui en ressortira sera sans nul doute celle d'une grande diversité. Thématiquement, il faudrait focaliser plus spécialement sur la perspective d'en bas ; au point de jonction entre État et peuple ; reconnaître les complexités de cette relation entre État et citoyens et ne pas les réduire à des relations entre un bourreau et des victimes ou vice versa ; intégrer l'épisode napoléonien dans un cadre chronologique plus large pour mieux comprendre le contexte dans lequel s'est imposée la domination napoléonienne ; éviter les grandes thèses sociologiques qui marginalisent la politique locale. Enfin, il serait utile de comparer l'Empire napoléonien avec d'autres États et d'autres empires, tels ceux qui mènent une politique d'expansion dans de nouveaux territoires à peu près au même moment : les Britanniques en Irlande et en Inde ; les Russes en Finlande, en Pologne et dans le Caucase ; les Autrichiens en Italie; les Prussiens en Allemagne occidentale, etc.

\section{Annie Jourdan}

4. Voilà de quoi occuper les chercheurs en histoire napoléonienne, et pour longtemps. Du reste et pour revenir aux observations de Steven sur Howard Brown, une rupture du point de vue pénal ou judiciaire (1802) ne veut pas dire une rupture du point de vue politique : la constitution de l'an VIII est là pour le prouver. Et du point de vue juridique et social, c'est encore tout autre chose. Les ruptures connaissent donc des temporalités décalées, dont il faudrait tenir compte.

Mais que savons-nous au juste des réalités "indigènes 》 et des relations quotidiennes entre " occupants » et «occupés»? À vous lire, j'ai l'impression que vous différez entre vous, du moins pour ce qui touche à l'attitude des Français dans les pays conquis ou alliés. Je pense évidemment au pragmatisme invoqué par Rowe dans plusieurs articles ; à l'impérialisme invoqué par Broers dans son dernier livre et à l'idéologie invoquée par d'autres. Sous quelle impulsion agissent les fonctionnaires et administrateurs français? Que pouvez-vous dire à ce sujet? 


\section{Michael Rowe}

Sur les relations occupants/occupés, ce sont les sources qui déterminent le travail. Les « révoltés » en général évitent de laisser des traces; et s'ils le font, ces documents ont été produits le plus souvent par des autorités qui leur sont peu acquises ou bien qui ont intérêt à minorer l'opposition. Il est évident qu'un préfet napoléonien dans un pays comme la Rhénanie sera bien avisé - en fonction de ses perspectives personnelles de carrière - de faire passer l'hostilité de l'opinion pour l'expression d'une minorité de moines fanatiques, nullement représentative des sentiments de l'élite locale... des gens dont le métier de préfet était justement de guider l'opinion!

Les historiens de cette période doivent envisager la « collaboration » et la « résistance » pour ce qu'elles sont, à savoir des extrêmes à chaque bout de l'éventail des comportements. Quelques-uns des meilleurs travaux - tel le livre récent de John Davis sur le Royaume de Naples - ont démontré combien un comportement qui, à un niveau, apparaît comme étant pure collaboration (accepter une charge publique à l'intérieur des institutions françaises) peut dissimuler une forme de résistance (saboter les réformes de l'intérieur). Le recueil publié récemment par Mark Philp sur la Grande-Bretagne à l'époque de la rumeur d'une descente de Napoléon a amplement prouvé quels étaient les motifs qui se cachaient derrière les invocations officielles d'un "patriotisme de défense nationale ». La majorité des Européens du début du XIX ${ }^{\mathrm{e}}$ siècle étaient des gens éclairés qui en général comprenaient que ce qui servait le mieux leurs intérêts n'était sûrement pas la résistance armée ou la collaboration pure et simple. Les historiens doivent l'être tout autant quand ils mettent au jour ces comportements.

À choisir, je me classerais moi-même comme un pragmatique. Peut-être que cela est dû au territoire que j'ai spécifiquement étudié, à savoir la Rhénanie. Un territoire clos au centre de l'empire napoléonien. Les fonctionnaires d'origine française qui y étaient détachés avaient moins de raison de s'y croire au cœur d'une « altérité ». C'est d'autant plus vrai que nombre d'entre eux étaient d'origine alsacienne, un groupe dont les facultés bilingues étaient exploitées par le gouvernement parisien, lequel les envoyait sur la rive gauche en nombre disproportionné. Historiquement, culturellement et géographiquement, ces hommes venaient essentiellement d'un même espace régional. Ils avaient plus de traits en commun avec les Rhénans qu'ils n'en avaient avec le Midi de la France.

Peut-être la division entre " pragmatiques 》 et "idéologues » reflète-t-elle une division Nord/Sud, avec une ligne de démarcation, tra- 
versant la France (comprenant l'Allemagne au nord, et l'Espagne, l'Italie et l'Illyrie au sud). Le pragmatisme en tant que réaction à l'arrogance impériale semble en effet être une réponse logique des États policiers, bien administrés du Nord, qui, n'en déplaise à la lettre si souvent citée de Napoléon à Jérôme n'avaient pas besoin de beaucoup de réformes pour fonctionner correctement.

Et pourquoi serions-nous surpris que les fonctionnaires impériaux approchent les situations différemment? L'uniformité administrative française a tenté d'en cacher les différences ; dans l'empire britannique, avec ses colonies, dominions, protectorats et pays sous mandat, elles étaient légion. On peut aussi invoquer un autre empire - celui de l'Allemagne nazie - qui était moins homogène et consistait en un agglomérat de gouvernements désunis, chacun ayant son agenda propre - certains pragmatiques ; d'autres plus idéologiques. Dans quelle mesure peut-on appliquer ce schéma à l'Europe napoléonienne ? Et puis, il ne faut pas négliger les changements qui s'opèrent au cours du temps : le pragmatisme distinguant la première génération des administrateurs (des individus qui avaient vécu les diverses étapes très chargées idéologiquement de la Révolution française et qui s'y étaient opposés à un moment ou à un autre) de la seconde génération - des jeunes auditeurs de vingt ans lors des dernières années de l'Empire (trop jeunes donc pour se souvenir de la Révolution), pour qui l'idéologie était importante, et ce en dépit d'une ascendance bien souvent noble.

\section{Michael Broers}

De nouveau, je ne me retrouve pas du tout dans ces observations. Rowe, moi-même, Esdaile, Grab et Davis, nous avons concentré beaucoup d'attention aux « occupés » et aux archives locales. Mais notons bien que la plupart d'entre elles sont des documents officiels, comme le rappelle Rowe. Or, ce sont de ces documents dont nous sommes dépendants pour apprendre ce que disent les " occupés »- notamment sur les révoltes populaires et les résistances, ce qui pose des problèmes fondamentaux aux historiens de la période. Deuxièmement, et j'en sais quelque chose, l'ironie du sort veut que ce soit dans les archives centrales ou nationales que l'on trouve le plus d'informations sur les localités : à Paris, Milan, Naples ou Kassel. En tout cas, telle que la question est posée, je ne suis pas d'accord: nombre de travaux ont été publiés et ces événements ont été bien étudiés.

Sur le second point, je crois que ce sera là le cœur du débat qui va s'ouvrir au cours des prochaines années dans le monde anglo-saxon. Il y 
a beaucoup à dire - chaque aspect a sa place, parce que chacun d'eux est présent à un moment ou à un autre. Ainsi l'imposition du Code, du Concordat et de l'administration risquait de problématiser les choses pour les Français. Cette politique rigide a rendu en effet la tâche (pragmatique) d'exploitation - conscription, séduction des élites en vue d'un soutien dans la levée d'hommes et d'argent - beaucoup plus difficile qu'elle n'aurait pu l'être.

\section{Steven Englund}

C'est un point qui dépasse mes compétences, mais je crois savoir qu'il y a des informations fiables sur les peuples occupés, même si elles sont loin d'être suffisantes. Broers a écrit sur le sujet, tout comme John Tone l'a fait sur les guérillas espagnoles et John Davis sur l'Italie méridionale. Alexander Grab a publié un formidable article sur les brigands et la révolte de 1809 en Italie septentrionale, où il utilise les archives judiciaires et examine les dépositions des villageois eux-mêmes. Enfin, Johan Joor a fait du bon travail en Hollande. Si sa thèse est en néerlandais, il a écrit quelques articles en anglais et en français sur le sujet.

Si j'en crois les discussions avec des historiens passionnés par le « vécu des indigènes », j'en conclus qu'il n'y a pas autant de documents pertinents dans les archives que ce qu'on pourrait penser ou espérer. La question des « révoltes » contre l'occupation française, quoi qu'il en soit, n'aboutira jamais au consensus, parce que c'est une question très controversée, tant par les contemporains que par les modernes. Il y en aura toujours pour croire, et pas seulement parmi les historiens français, que l'occupation et l'influence françaises étaient tout à la fois positives et négatives. C'est la nature même du Premier Empire que d'être ambigu. Malheur à qui l'oublie.

Pour ce qui est de la théorie post-moderne, telle qu'elle s'est développée dans l'historiographie des empires coloniaux des XIX et $\mathrm{XX}^{\mathrm{e}}$ siècles et de son application au cas du Premier Empire, j'ai déjà mis sur papier mon sentiment à cet égard (dans The Historical Journal) et critiqué les tentatives que fait Broers dans ce sens - aussi brillantes et originales qu'elles soient, elles sont profondément défectueuses, à mon avis. Mais j'ai aussi une position a priori sur le débat entre pragmatisme versus idéologie. L'idéologie était certainement un élément de poids dans la manière de gouverner l'empire napoléonien, mais il y en avait d'autres tout aussi, sinon plus importants (extraction d'argent et d'hommes, imposition de l'ordre, etc.), selon le moment de cette politique et les hommes qui l'exécutaient. Alexander Grab a très bien défini à quel point idéologie 
et pragmatisme s'entremêlent dans son Napoleon and the Transformation of Europe (Palgrave, 2003), et il démontre une fois de plus combien il est difficile d'appliquer les subtiles distinctions analytiques des universitaires à une réalité combien plus complexe.

\section{Annie Jourdan}

5. Les lacunes des archives ont bon dos, si je puis me permettre. On s'en aperçoit dans les études locales, où les chercheurs découvrent des documents inédits qui éclairent l'attitude des populations - archives municipales, archives judiciaires et criminelles, archives militaires, etc. En tout cas, la thèse que je suis en train de lire sur un village du Limbourg néerlandophone où l'auteur décrit à loisir les péripéties des conscrits me donne une tout autre impression (J. Welten, In dienst voor Napoleons Europese droom, Louvain, 2007). Mais ce serait injuste que de vous le reprocher à vous qui aviez déjà tellement bien étudié les archives des pays impliqués.

Venons-en donc aux résistances. Qu'il y ait eu plus de révoltes populaires qu'on ne l'ait dit, ne signifie pourtant pas que les Français ont été confrontés à de véritables guerres "nationales 》 de libération ou à des guerres de "libération nationale ". C'est une réalité constatée aujourd'hui par bien de vos collègues que les révoltes d'Italie du Sud ou d'Espagne sont dues à des causes plus «triviales »: frustrations, opposition à la conscription, misère ou fiscalité. Mais du coup, cela semble infirmer la thèse " apocalyptique " de David Bell sur la guerre totale, laquelle aurait mis aux prises des " ennemis absolus ». S'il est question d'insurrections " circonstancielles » motivées par des frustrations passagères, on se demande pourquoi il serait utile d'invoquer de telles notions - créées du reste dans les circonstances bien différentes de l'Entre-deuxguerres allemand, ce qui pose la question de leur légitimité en matière d'explication historique.

\section{Michael Rowe}

Le consensus actuel est que le nationalisme n'était pas un facteur prédominant en Espagne après 1808, en Russie en 1812, en Allemagne en 1809-1813, ou encore en Grande-Bretagne entre 1803 et 1805 . Mais là se pose évidemment un problème spécifiquement sémantique : qu'entendons-nous par nationalisme ? Il n'y a pas jusqu'ici de définition consensuelle. Est-ce essentiellement la haine de l'Autre ? Dans quelle mesure le nationalisme doit-il être ou non socialement inclusif ? Doit-on y compter les «nationalismes nobles » de Hongrie et de Pologne? 
En fait, ce qui frappe, c'est que, dès qu'il s'agit de nationalisme, les recherches dans des domaines relativement proches vont dans des directions opposées. Les historiens politiques de l'Europe napoléonienne - qui regardent de haut en bas - conscients de leurs traditions historiographiques enracinées dans un terroir national, auxquelles ils tentent d'échapper, minorent tout à fait naturellement le nationalisme et soulignent à l'inverse les facteurs sociologiques. De là l'image que nous nous faisons du conflit, comme étant, semble-t-il, motivé par les querelles locales (entre ville et campagne, le plus souvent). Ainsi, il y aurait eu des résistances, mais elles n'auraient pas particulièrement été dirigées contre les Français parce qu'ils étaient français, mais parce qu'ils incarnaient l'État.

Par contre, les historiens qui se sont spécialisés dans l'étude de la sphère publique durant la période - ou plutôt durant une période qui englobe tout le dix-huitième siècle - soulignent le plus souvent que des segments importants du public européen de l'époque étaient remarquablement bien éduqués et informés en matière de politique. L'idée que nous aurions affaire à des mentalités " de clocher » serait tout à fait fausse. Qui plus est, quelques-uns des meilleurs ouvrages sur le nationalisme - Abigail Green sur l'Allemagne du XIX ${ }^{\mathrm{e}}$ siècle, par exemple - illustrent fort bien que le nationalisme est souvent imaginé à travers le local. Le local et le national ne sont pas incompatibles mais se renforcent mutuellement.

Traiter le nationalisme comme quelque chose de potentiellement dangereux durant la période napoléonienne n'est pas anachronique; ce n'est pas tomber dans un piège que d'utiliser rétrospectivement des concepts actuels. Car des gens - une minorité, certes, mais une minorité influente - pensaient en ces termes. Et les Français qui devaient prendre des décisions en 1813-1814 en étaient conscients, même s'ils ne parlaient pas à ce propos de nationalisme. Ils avaient peur que les affinités culturelles et linguistiques des Rhénans avec les Bavarois, les Autrichiens et les Prussiens n'aliènent les premiers de la France. Ces craintes étaient peut-être exagérées - il n'y a pas eu de soulèvement anti-napoléonien en Rhénanie entre 1813 et 1814 - mais elles ont influé sur la politique française : notamment le refus de mobiliser la garde nationale dans les départements « réunis » ou bien la décision de désarmer les régiments néerlandais de l'armée impériale. La crainte du nationalisme - qu'elle soit ou non justifiée - a conduit les fonctionnaires français à agir comme s'ils étaient en territoires ennemis et à les évacuer prématurément - attitude qui n'a évidemment pas eu d'équivalent dans l'ancienne France. Dans quelle mesure cela a-t-il contribué à la défaite ultime de Napoléon? Peu, sans doute, étant donné l'ampleur de la coalition. Il n'empêche, les 
expériences des autres échecs - Prusse 1806, France 1940 - montrent bien que le moral et l'état d'âme sont des facteurs à prendre en considération. Les gouvernements autres que la France pensaient que les guerres napoléoniennes étaient aussi une "guerre d'opinion 》 (pour citer un contemporain allemand) : des guerres où l'opinion publique était importante. C'est même vrai en Russie, où la noblesse francophobe (soudée par sa haine de la Pologne et du catholicisme) a influé sur les prises de décision d'Alexandre. Le livre de Wolfgang Piereth sur la police du gouvernement bavarois en matière de presse allemande prouve que même les petits États ont investi du temps et de l'argent pour influer sur l'opinion. $\mathrm{Vu}$ de loin, on peut conclure que le nationalisme n'était pas généralement répandu, mais les gouvernements de l'époque n'en étaient pas certains et ont agi comme s'il l'était vraiment.

Il faut être prudent sur ce qui est de la guerre totale, laquelle remémore les images de Goya, figurant des victimes massacrées sans état d'âme : un genre d'activité très différente du professionnalisme détaché, caractéristique de la guerre du dix-huitième siècle. Total ou totale implique une volonté inflexible de détruire complètement l'ennemi. La question posée semble par ailleurs suggérer que se battre pour des motifs essentiellement locaux (contrairement à nationaux) ne saurait être «total». On peut rétorquer l'inverse : les conflits locaux, mettant face à face des voisins, sont les plus terribles et débouchent bien souvent sur une destruction complète de l'ennemi (y compris sa famille et ses biens). Mais ici encore, cela nous ramène aux commentaires sur le nationalisme, et sur sa force quand il dérive d'une imagination locale (tout à fait banale). Les paysans rhénans, qui, fin 1813, répondent à l'effondrement du régime français en s'en prenant aux gardes forestiers et aux gardes-chasses croyaient vraiment agir en patriotes allemands, ainsi qu'ils le disaient. Le fait est que les autorités prussiennes n'en croyaient rien et qu'elles les jetèrent en prison. En Espagne, il n'y avait pas d'autorités équivalentes à celles des Prussiens dans la Rhénanie de 1814. Là, c'est le chaos qui prévalait. En Russie, le maire de Moscou - Rostopchine - pensait apparemment que les masses russes étaient comme une bête incontrôlable, qui raserait tout sur son passage : sa réponse fut de s'assurer que ce serait l'armée française et non la noblesse russe qui en subirait les effets. Il y a deux variables ici : 1. l'intensité avec laquelle l'opposition locale s'était sentie oppressée par l'exploitation ennemie (française) - en Espagne, Prusse et Russie, c'était une cause importante dans certaines régions ; en Rhénanie, c'était moins perceptible. 2. l'intensité avec laquelle les autorités étatiques souhaitaient et étaient en mesure de réprimer ou d'encourager la fureur populaire : en 
Prusse, l'État était assez puissant pour faire en sorte que les choses n'échappent pas à son contrôle (et ceux qui étaient pour la guerre totale, comme Gneisenau, n'étaient pas écoutés du roi et de la plupart du corps militaire professionnel) ; en Russie et en Espagne, en revanche, même s'il l'avait voulu, l'État n'était pas assez puissant pour empêcher les excès. Tout ce qu'il pouvait faire, c'était orienter la fureur populaire directement vers les Français.

\section{Michael Broers}

En tant que groupe, je ne crois pas que nous acceptions l'idée de « guerres nationales de libération ». Moi, en tout cas, je n'y crois pas et je l'ai écrit plus d'une fois. Ceux que j'ai lus, non plus, ce me semble. Il est vrai qu'on a trop souvent eu tendance à suggérer qu'à tous les stades, Napoléon a subi des résistances au niveau local, particulier, «réactionnaire ». Le second problème soulevé à propos des catégories de pensée ou des instruments de classification est très intéressant, en effet. Mes vues ne sont pas encore fixées là-dessus, mais elles ont été stimulées par le livre de David Bell. Quand il s'agit de conflits locaux, je crois pourtant que nous pouvons penser en termes « d'ennemi absolu » ou « d'hostilité absolue », tels ceux de Carl Schmitt, sans les projeter pour autant sur la guerre conventionnelle. Je crois que l'hostilité absolue entre les guérillas française et espagnole ou bien avec les rebelles de Calabre n'a pas besoin d'être entièrement «politisée » selon des normes modernes. Elle peut résulter de la faim, de la conscription, de l'exploitation, etc. aussi bien que de facteurs idéologiques. Guerre totale et hostilité absolue ne sont pas synonymes, en termes concrets. L'une est une approche logistique et politique de la guerre conventionnelle; l'autre est une attitude, un état d'esprit. L'hostilité absolue n'a pas non plus besoin d'être aiguisée par la haute politique, plutôt l'inverse.

Cela me mène à mon dernier point : je crois que le concept de Carl Schmitt peut avoir une signification réelle - pas n'importe où et pas dans n'importe quelles circonstances. Je pense que celle-ci est plus spécifiquement le fait des intellectuels et des marginaux politiques d'une part, et de la guérilla de l'autre. Pour conclure sur le problème de l'anachronisme, mais sur un autre sujet: dans les nombreux travaux sur la position des femmes de l'époque et sur le divorce tel qu'il est décrit dans le Code, trop de préoccupations modernes envahissent les questions de gender - il y a un tas de sources qui indiquent que les femmes napoléoniennes se percevaient comme les plus libérées et les plus privilégiées de l'Europe d'alors. 


\section{Steven Englund}

Le problème des «guerres de libération nationale » a été depuis plus ou moins longtemps résolu par quelques spécialistes des pays concernés (Hartley, Hagemann, Esdaile, Davis, etc.), qui se retrouvent pour nier que le terme serait adéquat pour décrire ce qui était arrivé. David Bell résume ce consensus, quand il écrit, " les guérillas ne représentent pas en fait l'ensemble des populations - dans beaucoup de pays, Napoléon recevait un soutien significatif. Elles n'exprimaient pas toujours un patriotisme égoïste et leurs acteurs ressemblaient souvent à de vrais brigands plus que ne veulent l'admettre leurs admirateurs modernes. Elles cherchaient à mobiliser et à attaquer là où le régime français était le plus précaire, et non le plus oppressif [...]» (p. 266).

Selon moi, toute la question des guerres de libération nationale est un autre point qui démontre combien le débat souffre d'une surdétermination typiquement universitaire, due au flou qui entoure des termes ou des mots du genre « national » et « guerre totale ». Ces mots ne sont pas des termes rigoureux, comme « poids nucléaire » en physique. Au contraire, ce sont des concepts éminemment controversés. Ce qui se développe en fait en Allemagne, Italie, Espagne et Russie, ce sont des réactions violentes, plurivoques ou polysémiques, contre les occupants, qui acquièrent quelques traits de ce qu'on appellera plus tard les « guerres de libération nationale $»$. Ne pas le reconnaître serait en fait entreprendre une étude qui situerait les origines de la conscience nationale dans l'ère pré-moderne : les pré-conditions ne sont pas la chose en soi, mais juste des pré-conditions.

D'autre part, ce n'est pas parce que les hommes qui se rebellent contre l'Empire sont motivés par des problèmes « matériels » (conscription, impôts, pauvreté ou répression religieuse) que leurs révoltes sont « triviales ». Même les guerres coloniales du XXe siècle ont sans doute des causes «triviales ». Dans tout événement, Marc Bloch nous l'a appris, c'est une erreur que d'être hanté par la question des origines et leur supposée signification. On doit mesurer une série d'événements à leur évolution dans le temps, à leur ampleur, à leur dynamique générale. De ce point de vue, les révoltes espagnoles sont tout ce que l'on veut sauf « triviales » ou « circonstancielles ».

Loin d'être un théoricien inutile, le juriste Carl Schmitt a su saisir l'intensité des sentiments de haine et de ressentiment qui alimentaient nombre de rebelles dans les pays occupés. Ses catégories sur « l'Autre», «l'ennemi absolu », « la dictature souveraine ou la dictature de commissaire », «l'état d'exception » se sont développées pour une part à partir 
d'une étude sur les révoltes contre la France napoléonienne (et non à partir de la guerre conventionnelle confrontant des gouvernements, comme le note à juste titre Michael Broers). Ce sont des idées extrêmement importantes dont il faut tenir compte quand on étudie ce qui a vraiment provoqué la fureur et la violence sous le Premier Empire. Ce serait d'un moralisme suicidaire que d'éviter de lire et d'utiliser Schmitt simplement parce que nous n'aimons pas sa politique et que nous réprouvons ses actes sous le Troisième Reich. On n'est pas censé non plus aimer les idées de Marx, Weber, Durkheim ou Mannheim, mais on utilise leurs concepts.

D'un autre côté, il est maladroit d'appliquer sans plus des concepts contemporains sur les réalités du dix-huitième siècle. Il n'empêche. L'usage prudent d'anachronismes pour éclairer notre compréhension de l'histoire est précisément ce que l'historien professionnel est invité à faire, dès qu'il a établi et ordonné ses données empiriques. On n'attend pas de nous que, dans notre étude des périodes antérieures, nous adoptions leurs propres termes et leurs catégories de pensée - loin de là ! Et le voudrions-nous que nous ne le pourrions pas.

Tout cela me ramène à David Bell. J'ai profité de son livre en ce qu'il m'a incité à aller contre mes propres instincts et inclinations, et à me reconcentrer sur le métapolitique, au lieu de LA politique au jour le jour. Plus particulièrement, son livre jette un regard pénétrant sur l'élément décisif du métapolitique dans cette echt-politisches Epoch: à savoir, l'émergence d'une disposition culturelle en faveur d'une nouvelle forme de guerre, dont l'avènement s'est fait jour à la fin du XVIII' siècle, sans que les contemporains n'en aient pris conscience. Subrepticement, la guerre classique s'est transformée en une poursuite de buts vagues et illimités; a mobilisé de grands secteurs de la société, et parfois - pour un temps restreint - toute une société. En épousant sans le savoir ces nouvelles valeurs culturelles, la société française s'est «prussianisée », pour ainsi dire. Le secteur civil, même quand il restait dominant, s'est militarisé dans ses valeurs, ses modes, son style et souvent même jusque dans sa politique et ses grands fonctionnaires d'État. Selon Bell, Jean-Paul Bertaud aurait donc raison de voir dans la France napoléonienne une société nouvellement militarisée, nonobstant le fait que l'empereur et ses principaux collaborateurs demeurent pour la plupart civils et qu'ils s'évertuent à créer une société civile : "Bien que [Napoléon] n'ait pas gouverné par le militaire, il a gouverné dans une large mesure pour lui ». Voilà qui est plein de bon sens et spirituellement dit. La tragédie quasi grecque où se sont trouvés enfermés les Français (et certains de leurs alliés) entre 1805 et 1815 , alors qu'ils poursuivaient une guerre 
qu'ils ne pouvaient ni comprendre ni maitriser, c'est cette naissance d'une mentalité nouvelle et d'une définition inédite du conflit et de la violence. Elles les engloutissaient. Bell illustre le rôle nouveau de la guerre et du conflit tel qu'il s'est inscrit dans les esprits européens, en examinant le cas de Choderlos de Laclos et, quelques décades plus tard, celui de Stendhal et il montre bien combien l'idéologie contagieuse de la guerre en est venue à infecter la pensée des élites européennes. Il s'agissait désormais d'une partie de « quitte ou double », où l'enjeu était la victoire ou la défaite totale entre des adversaires devenus ennemis.

\section{Commentaires de conclusion}

Pour clore ce dossier, et dans la plus pure tradition anglo-saxonne, je me permettrai quelques commentaires sur plusieurs remarques des intervenants. Mais avant, il me faut les remercier de nous avoir donné un aperçu précieux des dialogues que nous pourrions avoir avec le monde historique anglo-américain.

Mon premier commentaire a trait aux remarques de Steven sur l'usage qu'on est en droit ou non de faire des concepts d'un auteur « politiquement incorrect ». Je voudrais rétorquer qu'il est pour le moins essentiel de s'interroger sur le bien-fondé de leur application à des contextes très différents - tels que les XVIII ${ }^{\mathrm{e}}$ et $\mathrm{XIX}^{\mathrm{e}}$ siècles. Par ailleurs, Schmitt distingue clairement entre deux sortes de guerres : la guerreaction et la guerre-état - dans la traduction française, qui, espérons-le, ne diffère pas de sa version anglaise. Dans la guerre-action, il y a certes un ennemi, mais c'est un ennemi adversaire : un ennemi réel - et la guerre est elle-même réelle. Ce serait justement le cas en Espagne, où le partisan défend sa terre contre un ennemi réel (les Français) qu'il veut « bouter » hors de son pays, à l'instar de Jeanne d'Arc, citée explicitement dans ce contexte (C. Schmitt, Théorie du partisan, Champs Flammarion, 1992, p. 161, 299 et 302). Là, il y aurait limitation de l'hostilité - et c'est ce que j'entends par «trivial» : des causes passagères. «L'ennemi absolu », en revanche, serait « l'ennemi présupposé », celui de Lénine ou de Mao, qui impliquerait «l'existence d'un ennemi subsistant au-delà de la cessation des hostilités immédiates et violentes 》. Quant à la "guerre totale », Schmitt ajoute qu'elle tire son sens «d'une hostilité présupposée qui lui est conceptuellement antérieure ", et là encore, il n'en décèle aucune occurrence avant 1914-1918. C'est là un point éclairci par l'auteur luimême, qui permet de douter de leur pertinence pour la période qui nous concerne. Enfin, s'il est devenu impossible de repenser le XVIII avec ses 
propres catégories de pensée ainsi que le veut Steven, pourquoi serionsnous en mesure de le faire avec celles du début du XX $\mathrm{XX}^{\mathrm{e}}$ ? Celles-ci ne nous sont-elles pas à leur tour devenues inaccessibles et étrangères ? Ou bien le terrorisme actuel les a-t-il ressuscitées ? Car derrière ces discussions plane en permanence l'ombre de Ben Laden et du 11 septembre.

Par contre, je suis pleinement d'accord avec Steven quand il souligne que l'époque pose seules les bases pour la naissance d'une conscience nationale et qu'il s'agit de pré-conditions. Mais du coup, j'aurais envie de raisonner de la sorte pour ce qui est du concept éminemment controversé de " guerre totale ", si bien que les guerres révolutionnaires et impériales seraient tout au plus des « guerres pré-totales ». Quant au problème des origines, il y a plus longtemps que ce concept m'indispose - car « origine » tient de l'ordre ontologique, qui n'est pas l'apanage de l'historien. Ce n'était pas non plus la question. Celle-ci concernait plus précisément les conclusions récentes des historiens anglo-saxons qui nient justement qu'un nationalisme ait été à l'origine des révoltes et des résistances - du moins auprès des gens simples, puisqu'on sait que parmi les intellectuels allemands en revanche, l'humiliation ressentie donne naissance à un sentiment « national », ainsi que le souligne du reste M. Rowe. En vérité les hommes du XVIII ${ }^{e}$ pensaient en termes de caractères nationaux (compatibles ou incompatibles) - qui, eux, étaient depuis le XVII ${ }^{\mathrm{e}}$ siècle monnaie courante et permettaient de se situer par rapport à l'étranger. Ce sont ces catégories de pensée qui sont contemporaines aux révolutionnaires et aux impériaux.

Pour ce qui est de la militarisation de la France du Premier Empire, permettez-moi de douter tout au moins de son ampleur. Pour qui examine la politique culturelle et juridique de l'époque, ce qui frappe, c'est la volonté napoléonienne de reconstruire une société strictement civile liant civilisation et modernité. Le Code civil en témoigne plutôt bien, puisqu'il réglemente les relations sociales sur les bases (révolutionnaires) de l'égalité et de la propriété. Et cela n'a pas été conçu pour le militaire. La même chose vaut évidemment pour une part de la politique culturelle, dont le musée Napoléon est le symbole par excellence, mais aussi dans les écoles dont le contenu du moins demeure classique. En réalité, le Premier Empire connaît trois sphères : politique, militaire et civile, auxquelles participent plus ou moins la plupart des Français. Insister exclusivement sur le militaire fait violence à une réalité beaucoup plus complexe. De même, focaliser sur la répression n'éclaire qu'un volet du vaste diptyque que fut le règne de Napoléon. C'est ne mettre en lumière qu'une pièce du formidable puzzle qu'est le passé. Et de fait, les trois auteurs s'accordent pour estimer qu'il reste 
encore beaucoup à faire avant que ne soit découvert sous toutes ses facettes l'impact réel de la politique impériale sur le continent européen.

Au terme de ces discussions, il apparaît évident que ces éminents historiens anglo-américains, d'origine assez diversifiée pour le reste, se retrouvent surnombre de points, notamment dans leur perception de l'historiographie française, trop centrée sur la France et pas assez sur l'étranger, et même si Broers rappelle qu'il ne faut jamais généraliser; et dans leur certitude, aujourd'hui de plus en plus acceptée, qu'il est temps de délaisser l'histoire nationale au profit d'une histoire supranationale. Certes, Broers et Englund se séparent quant à l'interprétation du Premier Empire en tant que précurseur d'une idéologie impérialiste quelque peu raciste et, comme il va de soi, autoritairement civilisatrice. Ici, Englund refuse d'antidater une idéologie (coloniale), si particulière au XIX ${ }^{\mathrm{e}}$ siècle. Par contre, tous trois sont une fois de plus d'accord pour voir la nécessité en France d'une ouverture plus ample aux discussions internationales, et surtout, ils nous rappellent que le Premier Empire est un objet d'études qui dépasse le cadre strictement national. Napoléon fut un des premiers Européens modernes. Et tel il doit être étudié. Il n'est plus l'apanage des Français, si tant est qu'il l'ait jamais été.

Michael Broers

Université d'Oxford michael.broers@history.ox.ac.uk

Steven ENGLUND

Université Américaine de Paris amdgsle@aol.com

Michael Rowe

Kings College London michael.rowe@kcl.ac.uk

Annie JOURDAN

Université d'Amsterdam a.r.m.jourdan@uva.nl 
